\title{
Countable linear combinations of characters on commutative Banach algebras
}

\author{
J. F. Feinstein
}

\begin{abstract}
An elegant but elementary result of Wolff from 1921 ( $\mathbf{7})$, when interpreted in terms of Banach algebras, shows that it is possible to find a sequence of distinct characters $\phi_{n}$ on the disc algebra and an $\ell_{1}$ sequence of complex numbers $\lambda_{n}$, not all zero, such that $\sum_{n=1}^{\infty} \lambda_{n} \phi_{n}=0$. We observe that, even for general commutative, unital Banach algebras, this is not possible if the closure of the countable set of characters has no perfect subsets.
\end{abstract}

It is well-known that every finite set of distinct characters on a commutative Banach algebra is linearly independent. In this note we discuss related questions (and their answers) concerning countable linear combinations of characters.

Notation. Throughout this note, $\ell_{1}$ and $\ell_{\infty}$ will denote the usual spaces of sequences of complex numbers. For a Banach algebra $A$, we denote the character space of $A$ by $\Phi_{A}$.

We begin with the main question that we wish to discuss.

Question 1. Do there exist a commutative, unital Banach algebra A, a sequence of distinct characters $\left(\phi_{n}\right) \subseteq \Phi_{A}$ and a sequence $\left(\lambda_{n}\right) \in \ell_{1} \backslash\{0\}$, such that (with absolute operator norm convergence)

$$
\sum_{n=1}^{\infty} \lambda_{n} \phi_{n}=0 ?
$$

Suppose for the moment that we have found an example of such a commutative, unital Banach algebra $A$ and sequence of characters $\left(\phi_{n}\right) \subseteq \Phi_{A}$. Then, for distinct positive integers $m$ and $n$, the set $U_{m n}:=\left\{a \in A: \phi_{m}(a) \neq \phi_{n}(a)\right\}$ is a dense open subset of $A$. By the Baire category theorem, the intersection of all of these sets $U_{m n}$ is dense in $A$. In particular, there exists an $a \in A$ such that the complex numbers $\phi_{n}(a)(n \in \mathbb{N})$ are all distinct. Setting $\alpha_{n}=\phi_{n}(a)(n \in \mathbb{N})$, and considering the elements $a^{k} \in A(k \in \mathbb{N})$, we would then obtain a positive answer to the following question about complex sequences.

2000 Mathematics Subject Classification. Primary 46J10; Secondary 46J15.

Key words and phrases. Commutative Banach algebras, characters. 
Question 2. Do there exist sequences $\left(\lambda_{n}\right) \in \ell_{1} \backslash\{0\}$ and $\left(\alpha_{n}\right) \in \ell_{\infty}$ with all the $\alpha_{n}$ distinct and such that

$$
\sum_{n=1}^{\infty} \lambda_{n} \alpha_{n}{ }^{k}=0(k=0,1,2, \ldots) ?
$$

Conversely, given a positive answer to Question 2, we may obtain a uniform algebra $A$ and a sequence of characters on $A$ satisfying the conditions of Question 1. Indeed, by scaling, we may assume that we have sequences $\left(\lambda_{n}\right)$ and $\left(\alpha_{n}\right)$ satisfying the conditions of Question 2 and such that $\left|\alpha_{n}\right|<1$ for all $n \in \mathbb{N}$. Take $A$ to be the disc algebra, and let $\phi_{n}$ be evaluation at the point $\alpha_{n}$ of the open unit disc. Since the polynomials are dense in the disc algebra, it follows easily that the conditions of Question 1 are satisfied.

Question 2 is discussed in some detail in 2. They first observe that (1) is equivalent to each of the following two conditions:

$$
\begin{gathered}
\sum_{n=1}^{\infty} \lambda_{n} \exp \left(\alpha_{n} z\right)=0(z \in \mathbb{C}) \\
\sum_{n=1}^{\infty} \frac{\lambda_{n}}{z-\alpha_{n}}=0\left(z \in \mathbb{C},|z|>\sup _{n}\left|\alpha_{n}\right|\right) .
\end{gathered}
$$

The answer to Question 2 is yes. Indeed, as noted in [2], the first example of such a pair of sequences $\left(\lambda_{n}\right),\left(\alpha_{n}\right)$ (satisfying (3)) was found by Wolff in [7]. His example is so elementary and elegant that we include it here, along with some extra details.

Notation. Let $a \in \mathbb{C}$ and let $r>0$. Then we denote by $\Delta(a, r), \bar{\Delta}(a, r)$ respectively the open disc and the closed disc in $\mathbb{C}$ with centre at $a$ and radius $r$. We denote Lebesgue area measure on $\mathbb{C}$ by $m$.

ExAmple 3. [7] Let $D_{1}$ be the closed unit disc in $\mathbb{C}, \bar{\Delta}(0,1)$. We may choose sequences $\left(\alpha_{n}\right)_{n=2}^{\infty} \subseteq \Delta(0,1) \backslash\{0\}$ and $r_{n}>0$ such that the closed $\operatorname{discs} \bar{\Delta}\left(\alpha_{n}, r_{n}\right)$ are pairwise disjoint subsets of $\Delta(0,1)$, and such that

$$
m\left(D_{1} \backslash \bigcup_{n=2}^{\infty} \bar{\Delta}\left(\alpha_{n}, r_{n}\right)\right)=0 .
$$

Set $\alpha_{1}=0$ and, for $n=2,3, \ldots$, set $D_{n}=\bar{\Delta}\left(\alpha_{n}, r_{n}\right)$. Set $\lambda_{1}=-\pi=-m\left(D_{1}\right)$ and, for $n=2,3, \ldots$ set $\lambda_{n}=\pi r_{n}{ }^{2}=m\left(D_{n}\right)$, so that

$$
\sum_{n=1}^{\infty}\left|\lambda_{n}\right|=2 \pi<\infty
$$

Then it is clear that, for any complex-valued function $f$ which is harmonic on a neighbourhood of $D_{1}$, we have

$$
\pi f\left(\alpha_{1}\right)=\sum_{n=2}^{\infty} \pi r_{n}^{2} f\left(\alpha_{n}\right)
$$

and so

$$
\sum_{n=1}^{\infty} \lambda_{n} f\left(\alpha_{n}\right)=0
$$


It is now easy to see that the sequences $\left(\alpha_{n}\right),\left(\lambda_{n}\right)$ have the required properties. To check (2), for example, let $z \in \mathbb{C}$. Set $f(w)=\exp (z w)$, so that $f$ is an entire function of $w$. Applying (4) to $f$ gives

$$
\sum_{n=1}^{\infty} \lambda_{n} \exp \left(\alpha_{n} z\right)=0
$$

as required.

Note that this shows that there is a non-zero annihilating measure for the disc algebra which is concentrated on a countable set, given by the countable linear combination of point masses

$$
\sum_{n=1}^{\infty} \lambda_{n} \delta_{\alpha_{n}}
$$

Also, as discussed earlier, the corresponding countable linear combination of distinct evaluation characters $\varepsilon_{\alpha_{n}}$ on the disc algebra is zero: with absolute convergence in operator norm,

$$
\sum_{n=1}^{\infty} \lambda_{n} \varepsilon_{\alpha_{n}}=0
$$

These characters are not in the Shilov boundary of the disc algebra: the F. and M. Riesz theorem ([5. Chapter II, 7.10]) shows that that would be impossible for the disc algebra. However, it is possible, in general, for all of the characters to be in the Shilov boundary. Indeed, the example above may be used to give such a sequence of characters for the well-known tomato can algebra (see [5. Chapter I, Exercise 12] or [3, 2-8]) of functions continuous on a solid cylinder, and analytic on one face. In this case, the Shilov boundary of the algebra is equal to its character space. This example is not essential. (The term essential was introduced in [1: see also [3, 2-8]). However, by using de Paepe's construction [4] in place of the tomato can algebra, we may obtain such an example where the uniform algebra is essential and where the Shilov boundary is again equal to the character space.

If some extra conditions are placed on the sequence $\left(\alpha_{n}\right)$ then no such sequence $\left(\lambda_{n}\right)$ can exist. For example, in [2], the following result appears, which is an elementary consequence of the standard theory of polynomial approximation.

Proposition 4. Let $\left(\alpha_{n}\right)$ be a bounded sequence of distinct complex numbers. Suppose that the closure in $\mathbb{C}$ of $\left\{\alpha_{1}, \alpha_{2}, \ldots\right\}$ has no interior, and does not separate the plane. Then there is no sequence $\left(\lambda_{n}\right) \in \ell_{1} \backslash\{0\}$ satisfying (1).

There are some more general results in $\mathbf{2}$. For example, the authors give a full characterisation of those sequences of distinct complex numbers $\left(\alpha_{n}\right) \subseteq \Delta(0,1)$ for which there is a sequence $\left(\lambda_{n}\right) \in \ell_{1} \backslash\{0\}$ satisfying (1), under the further assumption that the sequence $\left(\alpha_{n}\right)$ has no interior limit points in $\Delta(0,1)$. Such a sequence $\left(\lambda_{n}\right)$ then exists if and only if almost every point of the unit circle is a non-tangential limit of a subsequence of $\left(\alpha_{n}\right)$. They also showed that this is equivalent to the requirement that the sequence $\left(\alpha_{n}\right)$ is a dominating sequence, i.e., every bounded analytic function $f$ on the unit disc satisfies

$$
\sup _{z \in \Delta}|f(z)|=\sup _{n}\left|f\left(\alpha_{n}\right)\right|
$$


Proposition 4 suggests that if some extra conditions are placed on either the sequence or the algebra in Question 1, then the answer may change. For example, Proposition 4 may be used, along with a Baire category theorem argument, to prove the following result. We shall not include the elementary proof, since the result also follows from the more general result (Theorem 9) below.

TheOREm 5. Let $A$ be a commutative, unital Banach algebra, and let $\left(\phi_{n}\right)$ be a sequence of distinct characters on $A$ such that $\left\{\phi_{1}, \phi_{2}, \ldots\right\}$ is a compact subset of $\Phi_{A}$. Suppose that $\left(\lambda_{n}\right) \in \ell_{1}$, and that (with absolute operator norm convergence)

$$
\sum_{n=1}^{\infty} \lambda_{n} \phi_{n}=0 \text {. }
$$

Then all of the $\lambda_{n}$ must be zero.

An alternative approach that yields rather more is to use the following easy lemma.

LEMma 6. Suppose that $A,\left(\lambda_{n}\right)$ and $\left(\phi_{n}\right)$ satisfy the conditions of Question 1. Let $E$ be the closure in $\Phi_{A}$ of $\left\{\phi_{1}, \phi_{2}, \ldots\right\}$. Let $B$ be the uniform closure in $C(E)$ of the restriction to $E$ of the Gelfand transform of $A$. Then $B$ is a non-trivial uniform algebra on $E$.

Proof. Clearly $B$ is a uniform algebra on $E$, and $B$ is annihilated by the non-zero, regular Borel measure $\mu$ on $E$ defined by

$$
\mu=\sum_{n=1}^{\infty} \lambda_{n} \delta_{\phi_{n}} .
$$

The result follows immediately.

This is more than enough to resolve Question 1 for one obvious restricted class of Banach algebras.

Corollary 7. Let $A$ be a commutative, unital Banach algebra such that the Gelfand transform $\hat{A}$ is self-adjoint. Then there are no sequences $\left(\lambda_{n}\right),\left(\phi_{n}\right)$ satisfying the conditions of Question 1.

Proof. The result follows immediately from Lemma 6 and the Stone-Weierstrass theorem.

We now return to conditions on the sequence of characters $\left(\phi_{n}\right)$. To help us we quote the following result of Rudin.

Proposition 8. [6. Theorem 4]. Let $X$ be a compact space which has no perfect subsets. Then there are no non-trivial uniform algebras on $X$.

We can now give a stronger version of Theorem 5 .

TheOrem 9. Let $A$ be a commutative, unital Banach algebra, and let $\left(\phi_{n}\right)$ be a sequence of distinct characters on $A$ such that the closure in $\Phi_{A}$ of $\left\{\phi_{1}, \phi_{2}, \ldots\right\}$ has no perfect subsets. Suppose that $\left(\lambda_{n}\right) \in \ell_{1}$ is such that (with absolute operator norm convergence)

$$
\sum_{n=1}^{\infty} \lambda_{n} \phi_{n}=0 .
$$


Then all of the $\lambda_{n}$ must be zero.

Proof. The result follows immediately from Lemma 6 and Proposition 8.

Note that, above, we have made heavy use of the fact the $\left(\lambda_{n}\right) \in \ell_{1}$, which enabled us to consider uniform algebras and annihilating measures. It is not at all clear what happens if this condition on $\left(\lambda_{n}\right)$ is removed, and some other form of convergence (such as conditional operator norm convergence or strong operator topology convergence) is used when considering the series $\sum_{n=1}^{\infty} \lambda_{n} \phi_{n}$. I do not know whether or not some or all of the results above still remain valid in such cases.

I am grateful to Milne Anderson and John Wermer for useful discussions.

\section{References}

[1] Bear, H.S., Complex function algebras, Trans. Amer. Math. Soc. 90 (1959), 383-393.

[2] Brown, L., Shields, A., Zeller, K., On absolutely convergent exponential sums, Trans. Amer. Math. Soc. 96 (1960), 162-183.

[3] Browder, A., Introduction to function algebras, W. A. Benjamin, Inc., New York-Amsterdam, 1969.

[4] de Paepe, P. J., Essential function algebras with large Silov boundary, Math. Scand. 43 (1978), $325-328$.

[5] Gamelin, T.W., Uniform Algebras, Prentice-Hall, Inc., Englewood Cliffs, N. J., 1969.

[6] Rudin, W., Continuous functions on compact spaces without perfect subsets. Proc. Amer. Math. Soc. 8 (1957), 39-42.

[7] Wolff, J., Sur les séries $\sum \frac{A_{k}}{z-\alpha_{k}}$. C. R. Acad. Sci. Paris 173 (1921), 1057-1058, 1327-1328. School of Mathematical Sciences, University of Nottingham, University Park, NotTingham, NG7 2RD, UK.

E-mail address: Joel.Feinstein@nottingham.ac.uk 